\title{
Does Scientific Evidence for the Use of Natural Products in the Treatment of Oral Candidiasis Exist? A Systematic Review
}

\author{
Gabriela Lacet Silva Ferreira, ${ }^{1}$ Ana Luíza Alves de Lima Pérez, ${ }^{1}$ Ítalo Martins Rocha, ${ }^{1,2}$ \\ Mayara Abreu Pinheiro, ${ }^{1}$ Ricardo Dias de Castro, ${ }^{1}$ \\ Hugo Lemes Carlo, ${ }^{1}$ Edeltrudes de Oliveira Lima, ${ }^{1}$ and Lúcio Roberto Castellano ${ }^{1,2}$ \\ ${ }^{1}$ Postgraduate Program in Dentistry, School of Dentistry, Universidade Federal da Paraíba, 58051-900 Joao Pessoa, PB, Brazil \\ ${ }^{2}$ Human Immunology Research and Education Group (GEPIH), Escola Técnica de Saúde da UFPB, Universidade Federal da Paraíba, \\ 58051-900 Joao Pessoa, PB, Brazil
}

Correspondence should be addressed to Gabriela Lacet Silva Ferreira; gabriela_lacet@hotmail.com

Received 27 November 2014; Accepted 12 March 2015

Academic Editor: Arndt Büssing

Copyright (C) 2015 Gabriela Lacet Silva Ferreira et al. This is an open access article distributed under the Creative Commons Attribution License, which permits unrestricted use, distribution, and reproduction in any medium, provided the original work is properly cited.

\begin{abstract}
In view of the limitations of antifungal agents used in the treatment of oral candidiasis and the wide variety of natural products that have been studied as treatment of this disease, this systematic literature review proposed to evaluate whether scientific evidence attesting to the efficacy of natural products in the treatment of this disease exists. A systematic search in PubMed, MEDLINE, SciELO, Lilacs, and Cochrane Library databases was accomplished using the associations among the keywords Candida albicans, phytotherapy, biological products, denture stomatitis, and oral candidiasis in both English and Portuguese. Four independent observers evaluated the methodological quality of the resulting articles. Three studies were included for detailed analysis and evaluated according to the analysis protocol based on the CONSORT (Consolidated Standards of Reporting Trials) 2010 statement. The tested products were different in all studies. Two studies mentioned random samples, but no study described the sample allocation. No study mentioned sample calculations, a prior pilot study, or examiner calibration, and only one trial reported sample losses. Differences between the tested products and the methodological designs among these studies did not allow the existence of scientific evidence related to the effectiveness of these products for the proposed subjects to be confirmed.
\end{abstract}

\section{Introduction}

Oral candidiasis, which is produced by yeast of the genus Candida, is the mucocutaneous mycosis present in the oral cavity [1]. Generally, oral candidiasis affects users of complete upper dentures and is called denture stomatitis. Denture stomatitis is characterized by the presence of edematous and erythematous mucosa beneath an area covered by the prosthesis $[2,3]$.

Candida albicans is the most important microorganism in the pathogenesis of candidiasis and is present in the normal flora of the oral cavity. However, the transition from normal mucosal conditions to a parasitism situation may occur when an imbalance between host and fungus arises, which can lead to the onset of the oral candidiasis. The predisposing factors for oral candidiasis and denture stomatitis include systemic diseases, immune deficiencies, reduced salivary flow, broadspectrum antibiotic usage, continuous prosthesis usage nightly, smoking, and poor oral and denture hygiene [4].

Although these diseases can be asymptomatic, some patients may experience discomfort such as swelling, pain, and burning sensations in the mouth [5], impairing the ingestion of liquids and food and, consequently, the quality of life of these patients [6]. Several commercially available antifungal agents are used to treat oral Candida infection, including nystatin, amphotericin B, clotrimazole, miconazole, itraconazole, fluconazole, and ketoconazole. However, despite their effectiveness, these drugs may produce adverse effects such as bitter taste, allergic reactions, and drug interactions $[2,3]$.

The development of natural products capable of clinical application is needed to create new strategies to control oral 
candidiasis because of the drawbacks and weaknesses of commercially available products. Natural products are promising therapeutic alternatives because they tend to display much smaller and lower intensity adverse reactions compared to allopathic drugs. Notably, the study of natural products can provide health professionals with alternative, feasible, and low-cost therapies for treating oral diseases [7].

Therefore, the use of medicinal plants and natural products for the treatment of these diseases has been extensively investigated; however, the scientific evidence from these studies has not yet been consolidated. Randomized clinical trials are the most suitable study design for providing evidence regarding the effects of an intervention study. However, the results of only one of these studies are not sufficient to clarify certain issues. In this sense, systematic reviews and meta-analyses are the most appropriate and current methods to summarize and synthesize evidence regarding the effectiveness and effects of interventions $[3,8]$.

Thus, the aim of this study was to use a systematic literature review to evaluate whether scientific evidence attesting to the efficacy of natural products in the treatment of oral candidiasis exists.

\section{Materials and Methods}

A systematic literature review was performed using the methodology proposed by Higgins and Green [9]. The screening and selection of articles adopted the following criteria.

Inclusion Criteria. We included studies in English, Spanish, and Portuguese that were randomized controlled trials and systematic reviews of all ages and both genders that examined products for use in dentistry based on natural substances with or without reduced clinical and/or microbiological signs and symptoms of oral candidiasis.

Exclusion Criteria. We excluded all studies that did not meet the inclusion criteria of this research that evaluated the associations of synthetic and natural products.

Search Strategies. The identification of articles was accomplished using a systematic search in the PubMed (National Library of Medicine), MEDLINE (International Literature on Health Sciences), SciELO (Scientific Electronic Library Online), Lilacs (Latin American and Caribbean Literature on Health Sciences), and Cochrane Library databases.

The search strategy in PubMed was performed based on the association of the following words using the search option "all fields": (Candida albicans AND phytotherapy) OR (Candida albicans AND biological products) OR (stomatitis, denture AND phytotherapy) OR (stomatitis, denture AND biological products) OR (candidiasis, oral AND phytotherapy) OR (candidiasis, oral AND biological products). To refine the search, filters such as controlled trial, systematic review, and humans were used.

The search for articles was performed such that the greatest number of studies was found. The strategy used in the MEDLINE (search option "subject descriptor"), SciELO (search option "subject"), Lilacs (search option "all indexes"), and Cochrane (search option "title, abstract, and keywords") databases was as follows: (Candida albicans AND phytotherapy) OR (Candida albicans AND biological products) OR (denture stomatitis AND phytotherapy) OR (denture stomatitis AND biological products) OR (oral candidiasis AND phytotherapy) OR (oral candidiasis AND biological products) OR (Candida albicans AND fitoterapia) OR (Candida albicans AND produtos biológicos) OR (estomatite sob prótese AND fitoterapia) OR (estomatite sob prótese AND produtos biológicos) OR (candidíase bucal AND fitoterapia) OR (candidíase bucal AND produtos biológicos).

All articles related to these word associations and published by May 2014 were selected for analysis. Four independent observers evaluated the methodological quality of the selected articles (the title and abstract) to verify whether these articles met the inclusion criteria. In cases where the data contained in the abstract were insufficient for determining the inclusion of the study, the full text was reviewed. After individual assessments, the examiners came to a consensus regarding the inclusion of studies for the evaluation of the full text.

Finally, the selected studies were screened using the Jadad scale [10], and those studies with scores greater than or equal to 3 were evaluated according to the analysis protocol based on the CONSORT (Consolidated Standards of Reporting Trials) 2010 statement [11].

Protocol followed by the examiners for the analysis of articles included in this systematic review is as follows:

(1) preliminary analysis: title, primary author, country, language, journal, impact factor, and year of publication;

(2) methodological review:

(2.1) primary outcome of interest: with or without reduced clinical and/or microbiological signs and symptoms of oral candidiasis;

(2.2) assessment of the quality of clinical trials: Jadad scale [10], with studies that obtained scores less than 3 being excluded from this review;

(2.3) methodological design;

(2.4) type of blinding and type of sample allocation;

(2.5) profile, sample size, and sample size calculation;

(2.6) loss of sample and reasons;

(2.7) masking of product color, smell, and taste;

(2.8) presence and characterization of placebo or control group;

(2.9) comparison between control and experimental groups at the beginning of the study: description of groups to assess the equivalence between them at the initial phase;

(2.10) quote of a pilot study;

(2.11) quality of result measurement: inter- and intraexaminer calibration;

(2.12) criteria used for clinical and/or microbiological evaluation for the disease diagnosis;

(2.13) statistical analysis and significance level; 
(2.14) type of clinical trial: phase I, II, III, or IV according to Chalmers et al. [12];

(3) analysis of intervention:

(3.1) pharmaceutical form of the test product: gel, paste, or mouthwash;

(3.2) product concentration;

(3.3) dose range: amount and frequency per day and the time when the product is being used;

(3.4) time of use (days or weeks);

(3.5) clinical condition assessment intervals;

(3.6) adherence to treatment, daily monitoring, and adverse effects (reports of discomfort caused by the product).

(4) analysis of results: verification of accuracy according to the confidence interval and the sample size;

(5) analysis of conclusions: determining whether conclusion meets the goals.

\section{Results}

According to the strategic search, 378 studies were found. After excluding repetitions, 301 different articles were identified (Figure 1). Of this total, fifteen articles met the inclusion criteria and were selected for further analysis. After careful analysis, three studies were considered of high importance and were included in this systematic review. The following three were the controlled clinical trials included in this analysis:

(i) treatment of oral thrush in HIV/AIDS patients using lemon juice, lemon grass (Cymbopogon citratus), and gentian violet [13];

(ii) comparison of the therapeutic effects of an aqueous garlic extract and a nystatin mouthwash on denture stomatitis [3];

(iii) miconazole gel compared with Zataria multiflora Boiss. gel in the treatment of denture stomatitis [2].

3.1. Data Description. The three trials were conducted in English $(n=3)$ in two countries, Iran $(n=2)$ and South Africa $(n=1)$. All three trials scored 3 on the Jadad scale $(n=3)$.

Table 1 presents data regarding the study design and characterization. All three trials were randomized $(n=3)$, and two were performed as a double-blind $(n=1)$ or tripleblind $(n=1)$ trial. The three studies described the sample profile; however, none of them mentioned the type of sample allocation.

One study reported follow-up losses; however, none of the studies mentioned conducting sample calculations or performing a pilot study or inter- and intraexaminer calibration. The three studies characterized the control group regarding the concentration of the product and the form of use. All articles provided the concentration of the test product, the quantity and time of use, and the intervals of clinical evaluation (Table 1).

Table 2 presents the data regarding the initial comparison between groups, the criteria used for the initial evaluation, and descriptions of the statistical analyses. All studies presented complaints of adverse effects and conclusions that corresponded to the study objectives (Table 2).

\section{Discussion}

Given the large amount of publications testing new products for clinical use, researchers, clinicians, and managers do not likely have access and time to evaluate all of these publications. Discerning and condensing all the information contained in these manuscripts to apply this knowledge to different clinical situations are even more difficult [14]. In this context, in dentistry, systematic reviews have been proposed for evaluating existing scientific evidence to respond to specific questions and to present the evidence in an accessible format.

The primary features of a systematic review are as follows: the creation of preestablished goals with inclusion and exclusion criteria for selecting studies, clear and reproducible methodology, systematic searches that provide access to the largest number of studies that meet the selection requirements, careful evaluation of the methodology and conclusions of the included studies, and organization and synthesis of results and conclusions [9] to minimize bias and to provide reliable results that support decision making [14].

Considering the limitations of commercially available antifungal agents for treating oral candidiasis, which involve increased fungal resistance [15], high cost, and adverse effects $[2,3]$ related to treatment, natural products have been investigated as important alternatives for the treatment of this pathology. The diversity of clinical and laboratory studies in the literature that have tested the different natural products raises this important question: is there clinical evidence for the use of natural products in the treatment of oral candidiasis?

To answer a clinical question, clinical trials are the studies of choice. The evaluation of a clinical trial includes careful methodological analyses of sample size, randomization, blinding, control usage, and sample losses [10].

The determination of sample size is an important part of the design of a clinical study because it attempts to eliminate both bias and predictable errors. A smaller sample than necessary can compromise the quality of the study, making understanding and inferring the results difficult; however, an extremely large sample may induce the existence of differences between groups when compared [16]. None of the studies included in this systematic review mentioned sample size calculations for sample determination.

The use of a control group is recommended to enable comparisons of test products preferably with the gold standard for treating the studied pathology. Thus, this review study included only controlled clinical trials.

Randomization and blinding are requirements cited in the literature [10] to assess the quality of clinical trials because randomization is a process in which each individual has the 


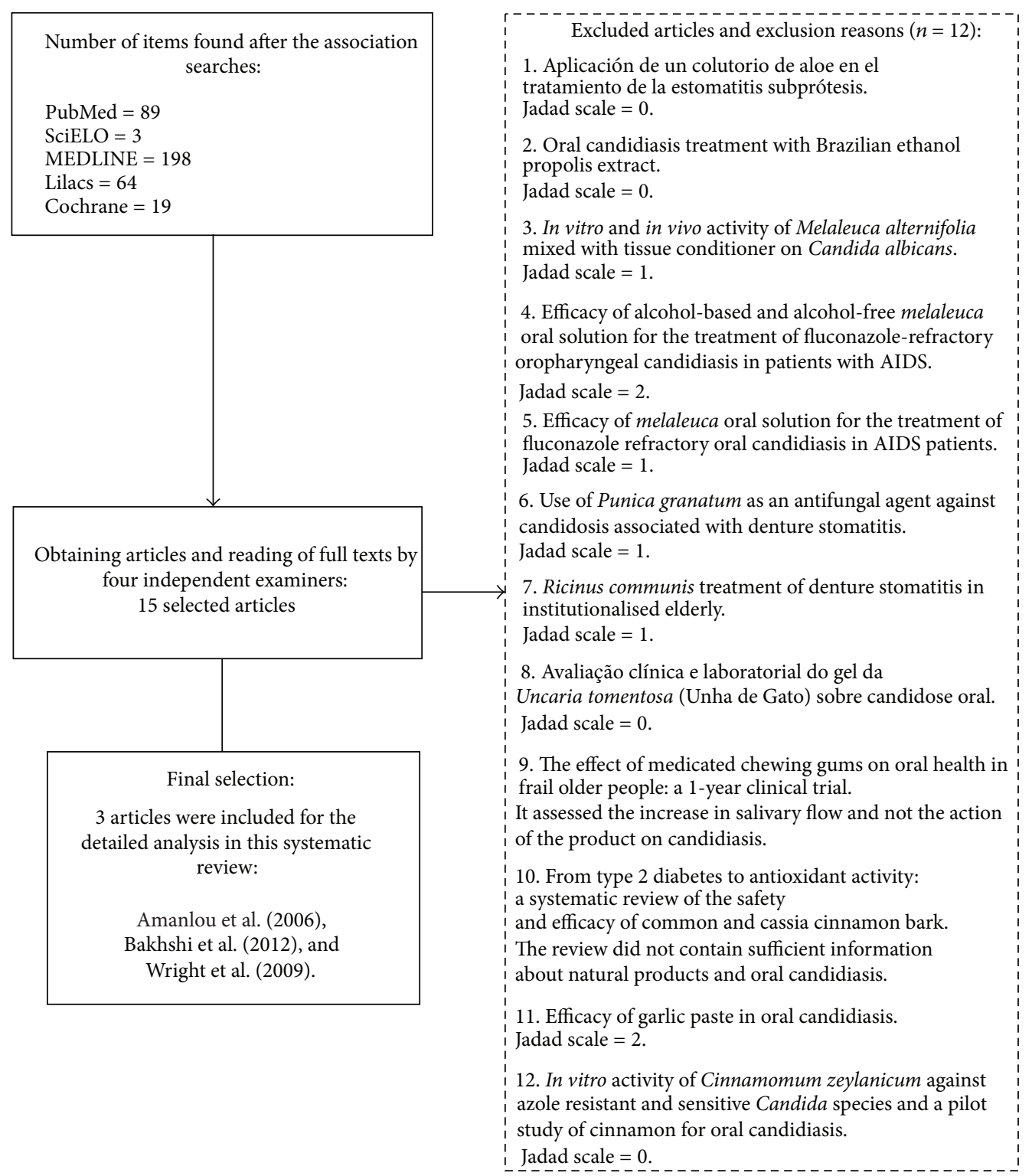

FIGURE 1: Flowchart of the search strategy.

same chance to participate in one of the groups. Blinding prevents the researcher, the individual, or the statistician to influence the results [11]. Both requirements prevent errors and biases during the study and were considered in the screening of manuscripts by applying the Jadad scale. Many studies were excluded because they did not mention randomization and blinding in the methodological procedures. Although most studies have indicated the use of these resources, Wright et al. [13] described the randomization process but did not perform blinding. Bakhshi et al. [3] described the process of randomization and blinding of researchers and statisticians involved; however, these authors did not detail the process of randomization and the masking of product color, taste, and smell, which would confirm the blinding of study participants. Amanlou et al. [2] characterized the blinding process, including the masking of product, but only mentioned the sample randomization process without describing it.

Because clinical studies involving monitoring participant follow-up for a certain period are subject to withdrawal due to several factors, losses are expected and should be mentioned [17]. Only Wright et al. [13] mention this fact. Because the greater the follow-up loss, the larger the questions regarding the study validity due to the higher occurrence of systematic errors [18], these data may be frequently omitted.

Another important aspect that should be considered in studies involving the follow-up of participants is maintaining a daily personal contact or media that try to encourage adherence to treatment and the correct use of products. None of the three included studies reported this form of contact with participants; however, in the study by Wright et al. [13], 


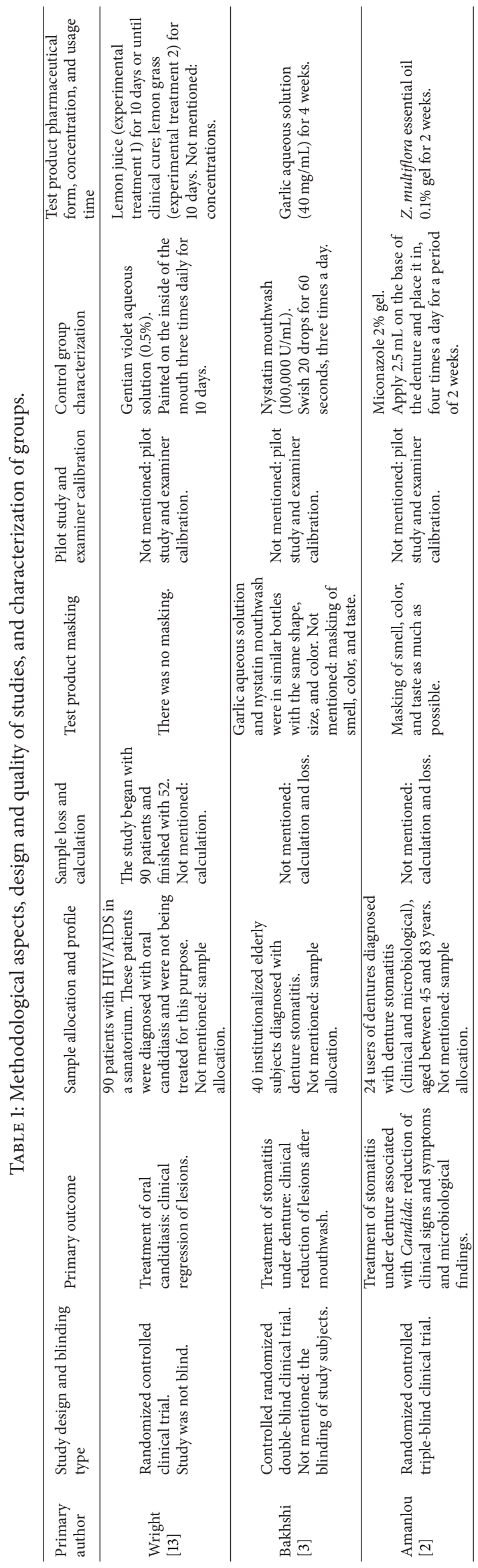




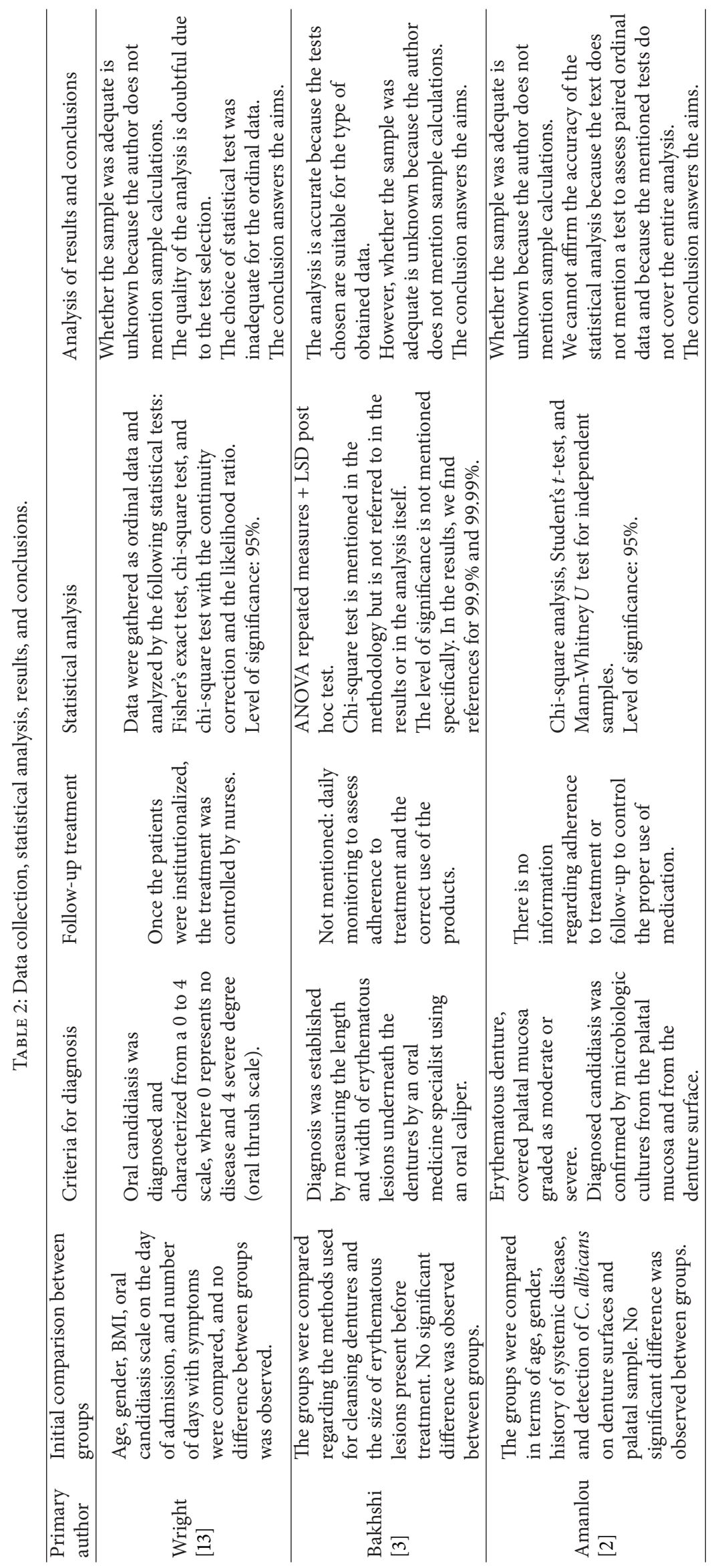


this form of contact was assumed to be controlled because subjects were institutionalized and because the products were administered by trained nurses.

The three evaluated studies showed different forms of intervention, including amounts, usage frequency, and treatment duration. This difference can be attributed to the different nature of the products tested and forms of presentation. During the intervention period and even after its completion, clinical evaluations to monitor treatment progress, as well as the presence of adverse effects observed by participants, are extremely important. All studies have reported followup intervals and recorded adverse effects. Amanlou et al. [2] and Bakhshi et al. [3] conducted weekly meetings with individuals for this purpose. Wright et al. [13] mentioned personal contact every two days. These follow-up intervals are relevant because the greater the proximity to subjects, the smaller the chances of follow-up losses and the greater the maintenance and effectiveness of the interventions.

Because clinical examination is of paramount importance in the diagnosis of oral candidiasis, microbial examination becomes an important auxiliary method for its confirmation. However, several diagnostic and classification tools for this disease are available, and, in an attempt to better understand and to compare data from different studies, these criteria should be standardized. All studies mentioned the stage of clinical examination but different parameters for the measurement and classification of lesions. Only Amanlou et al. [2] used the mycological exam of mucosa and dentures for confirmation.

Statistical analysis is an important step in analyzing the results because it reduces the probability of events occurring randomly. The judicious choice of the statistical test increases the reliability and accuracy of results [19]. One of the items evaluated in the selected studies was the use of statistical tests and their properties to provide answers to the guiding questions of the study according to the types of variables involved, the number of groups, and the sample size. Among the three studies evaluated, only Bakhshi et al. [3] clearly showed that the tests used were able to evaluate the data obtained and to answer the study objectives. However, all studies have limitations for not presenting a review of the clinical significance because the statistical significance presents the possibility of the obtained differences being true, regardless of the clinical importance, as determined by clinical judgment $[9,20]$.

A major difficulty in comparing the studies included in this systematic review was the variety of natural products tested and the forms of presenting these products. Confirming the existence of scientific evidence for the treatment of oral candidiasis with natural products is difficult when few studies meet the inclusion criteria and most have large methodological differences, whether in study design or in choosing the test product, concentrations, and pharmaceutical forms. All selected studies differ regarding these criteria. Thus, further clinical trials that address the study products by standardized methodologies and that evaluate different usage periods and various concentrations should be performed.

\section{Conclusion}

Currently, affirming the existence of scientific evidence for the use of natural products in the treatment of oral candidiasis is not possible.

\section{Conflict of Interests}

All authors declare that no conflict of interests regarding the publication of this paper exists.

\section{References}

[1] L. Coronado-Castellote and Y. Jiménez-Soriano, "Clinical and microbiological diagnosis of oral candidiasis," Journal of Clinical and Experimental Dentistry, vol. 5, no. 5, pp. 279-286, 2013.

[2] M. Amanlou, J. M. Beitollahi, S. Abdollahzadeh, and Z. Tohidast-Ekrad, "Miconazole gel compared with Zataria multiflora Boiss. gel in the treatment of denture stomatitis," Phytotherapy Research, vol. 20, no. 11, pp. 966-969, 2006.

[3] M. Bakhshi, J.-B. Taheri, S. B. Shabestari, A. Tanik, and R. Pahlevan, "Comparison of therapeutic effect of aqueous extract of garlic and nystatin mouthwash in denture stomatitis," Gerodontology, vol. 29, no. 2, pp. e680-e684, 2012.

[4] L. A. P. Pinelli, A. A. B. Montandon, S. C. T. Corbi, T. A. Moraes, and L. M. G. Fais, "Ricinus communis treatment of denture stomatitis in institutionalised elderly," Journal of Oral Rehabilitation, vol. 40, no. 5, pp. 375-380, 2013.

[5] L. C. de Souza Vasconcelos, M. C. C. Sampaio, F. C. Sampaio, and J. S. Higino, "Use of Punica granatum as an antifungal agent against candidosis associated with denture stomatitis," Mycoses, vol. 46, no. 5-6, pp. 192-196, 2003.

[6] J. A. Vasquez and A. A. Zawawi, "Efficacy of alcohol-based and alcohol-free melaleuca oral solution for the treatment of fluconazole-refractory oropharyngeal candidiasis in patients with AIDS," HIV Clinical Trials, vol. 3, no. 5, pp. 379-385, 2002.

[7] M. F. Vicente, A. Basilio, A. Cabello, and F. Peláez, "Microbial natural products as a source of antifungals," Clinical Microbiology and Infection, vol. 9, no. 1, pp. 15-32, 2003.

[8] S. Hasani-Ranjbar, B. Larijani, and M. Abdollahi, "A systematic review of the potential herbal sources of future drugs effective in oxidant-related diseases," Inflammation \& Allergy-Drug Targets, vol. 8, no. 1, pp. 2-10, 2009.

[9] J. P. T. Higgins and S. Green, Eds., Cochrane Handbook for Systematic Reviews of Interventions Version 5.1.0, The Cochrane Collaboration, 2011, http://www.cochrane-handbook.org/.

[10] A. R. Jadad, R. A. Moore, D. Carroll et al., "Assessing the quality of reports of randomized clinical trials: is blinding necessary?" Controlled Clinical Trials, vol. 17, no. 1, pp. 1-12, 1996.

[11] K. F. Schulz, D. G. Altman, and D. Moher, "CONSORT 2010 statement: updated guidelines for reporting parallel group randomized trials," Annals of Internal Medicine, vol. 152, no. 11, pp. 726-732, 2010.

[12] T. C. Chalmers, H. Smith Jr., B. Blackburn et al., "A method for assessing the quality of a randomized control trial," Controlled Clinical Trials, vol. 2, no. 1, pp. 31-49, 1981.

[13] S. C. Wright, J. E. Maree, and M. Sibanyoni, "Treatment of oral thrush in HIV/AIDS patients with lemon juice and lemon grass (Cymbopogon citratus) and gentian violet," Phytomedicine, vol. 16, no. 2-3, pp. 118-124, 2009. 
[14] L. Manchikanti, "Evidence-based medicine, systematic reviews, and guidelines in interventional pain management, part I: introduction and general considerations," Pain Physician, vol. 11, no. 2, pp. 161-186, 2008.

[15] R. D. Cannon, E. Lamping, A. R. Holmes et al., "Efflux-mediated antifungal drug resistance," Clinical Microbiology Reviews, vol. 22, no. 2, pp. 291-321, 2009.

[16] T. V. Macfarlane, "Sample size determination for research projects," Journal of Orthodontics, vol. 30, no. 2, pp. 99-100, 2003.

[17] E. Vervölgyi, M. Kromp, G. Skipka, R. Bender, and T. Kaiser, "Reporting of loss to follow-up information in randomised controlled trials with time-to-event outcomes: a literature survey," BMC Medical Research Methodology, vol. 11, article 130, 2011.

[18] J. Dettori, "Loss to follow-up," Evidence-Based Spine-Care Journal, vol. 2, no. 1, pp. 7-10, 2011.

[19] S. Wassertheil-Smoller and M. Y. Kim, "Statistical analysis of clinical trials," Seminars in Nuclear Medicine, vol. 40, no. 5, pp. 357-363, 2010.

[20] A.-W. Chan and D. G. Altman, "Epidemiology and reporting of randomised trials published in PubMed journals," The Lancet, vol. 365, no. 9465, pp. 1159-1162, 2005. 


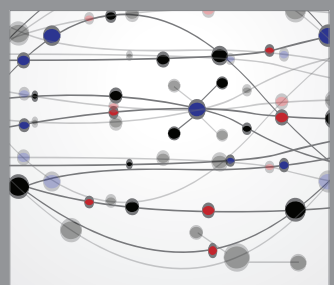

The Scientific World Journal
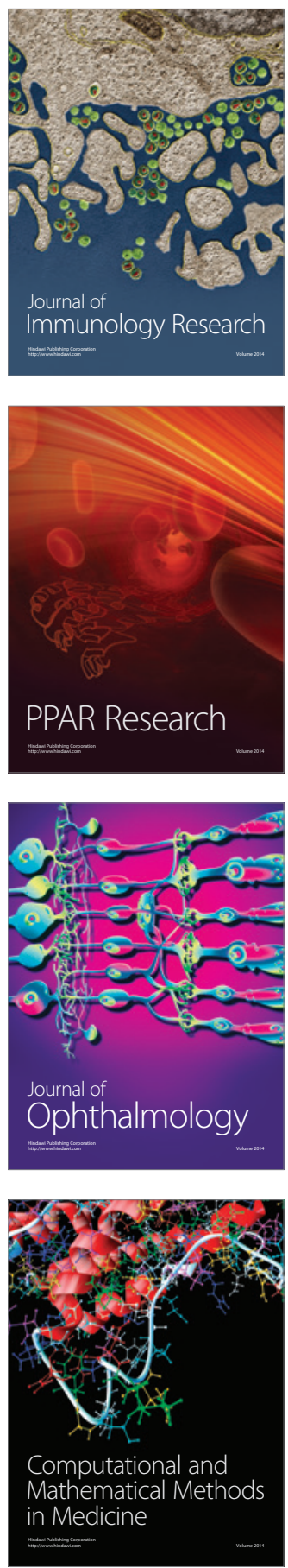

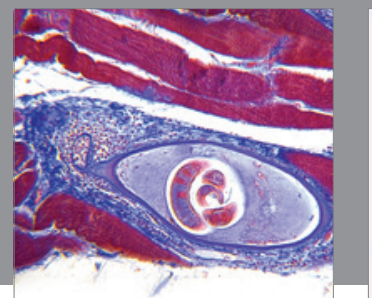

Gastroenterology

Research and Practice
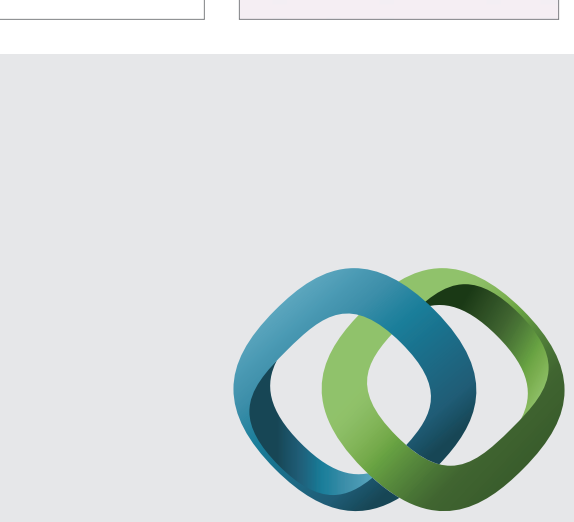

\section{Hindawi}

Submit your manuscripts at

http://www.hindawi.com
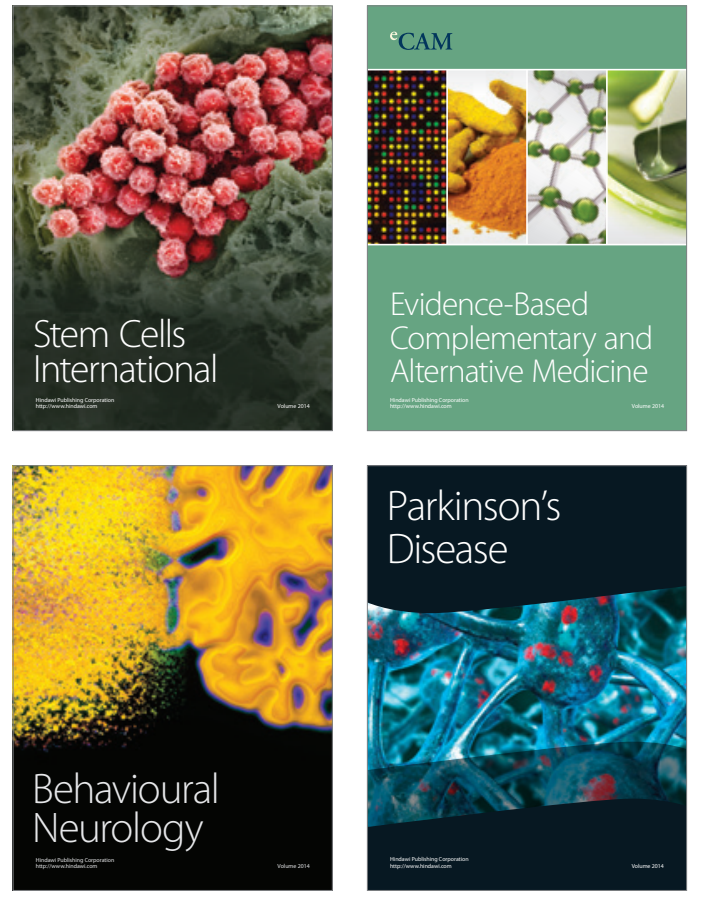
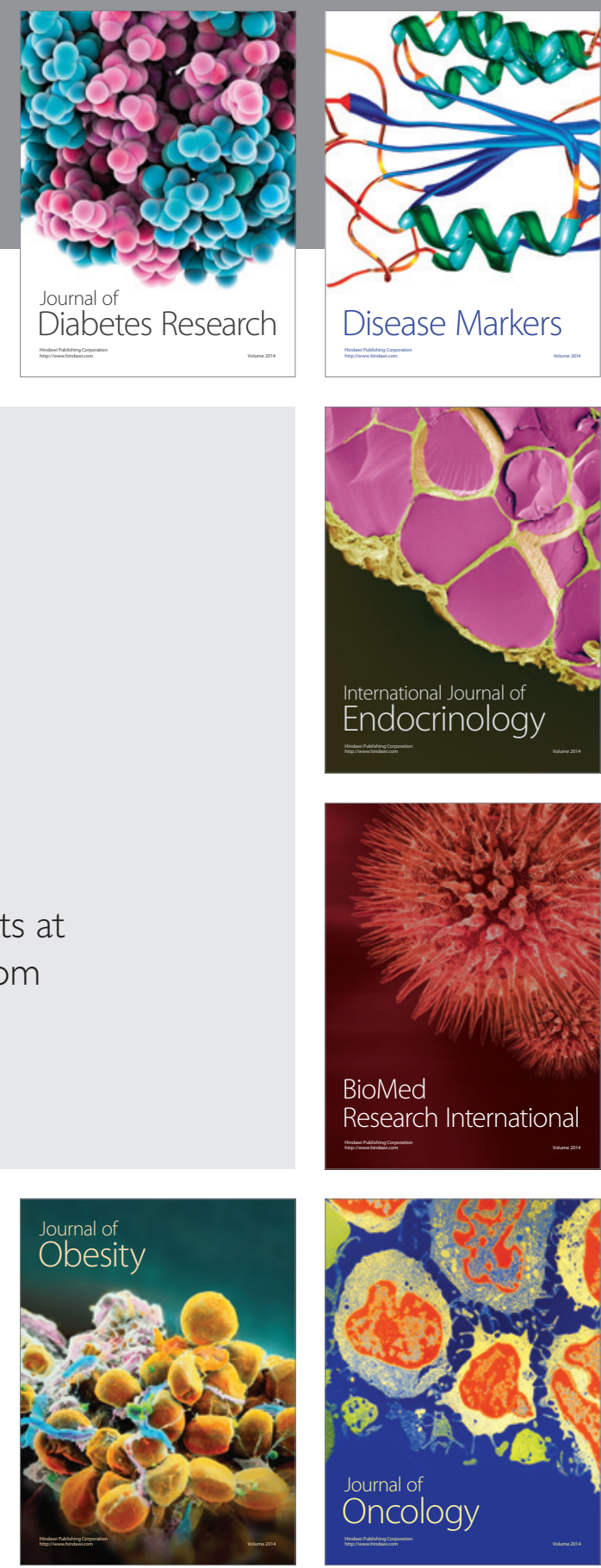

Disease Markers
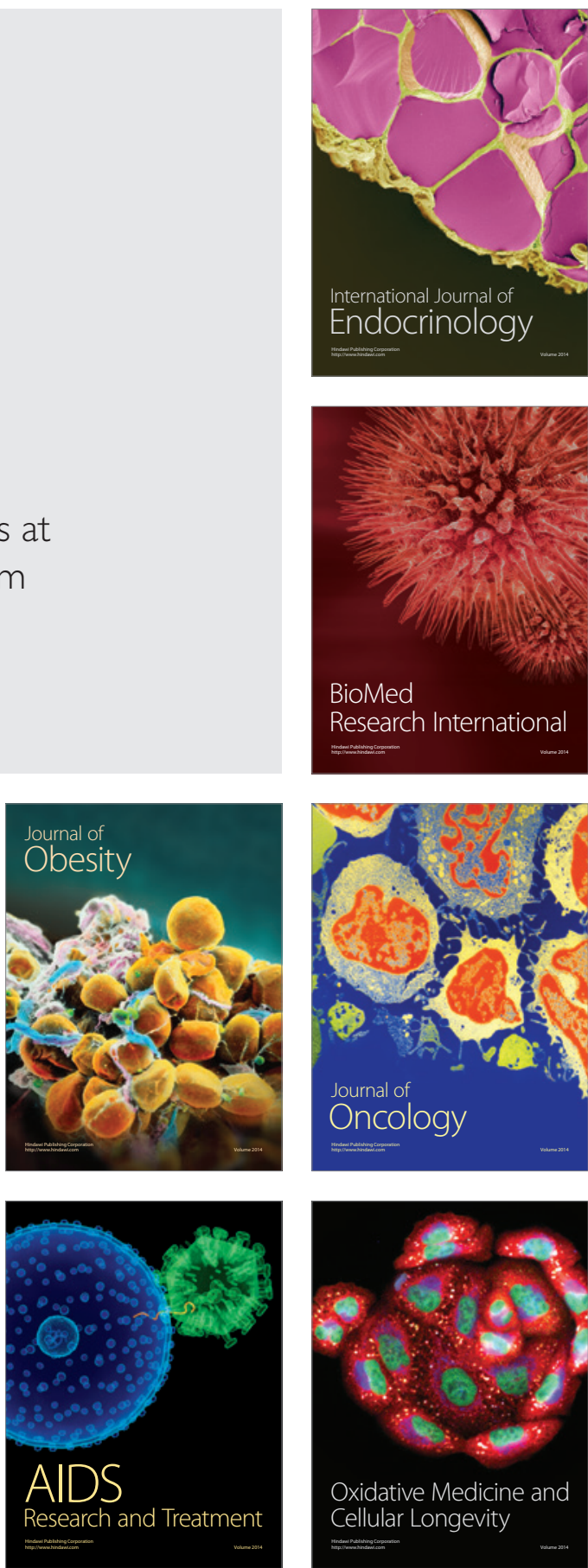